Sportwiss 2012 · 42:83-95

DOI 10.1007/s12662-012-0251-y

Online publiziert: 13. Juni 2012
Stefan Künzell ${ }^{1}$ Ernst-Joachim Hossner ${ }^{2}$

${ }^{1}$ Institut für Sportwissenschaft, Universität Augsburg

2 Institut für Sportwissenschaft, Universität Bern

\title{
Differenzielles Lehren und Lernen: eine Kritik
}

Und die Kammerherren gingen und trugen die Schleppe, die gar nicht da war.

Hans-Christian Andersen (1805-1875)

In den vergangenen 12 Jahren wurde von Wolfgang Schöllhorn und Mitarbeiter/ -innen das Konzept des differenziellen Lehrens und Lernens als Methodik für das Bewegungslernen entwickelt. Differenzielles Lernen (DL) ist charakterisiert durch „ständig wechselnde Bewegungsausführungen, Vermeiden von Wiederholungen, keine Fehlerkorrekturen [und] Realisieren von möglichst vielen Randbedingungen, die in klassischer Terminologie vor allem das Realisieren von Bewegungsfehlern beinhaltet" (Schöllhorn, Sechelmann, Trockel \& Westers, 2004, S. 15). ${ }^{1}$ Seit seiner erstmaligen Vorstellung (Schöllhorn, 1999) findet sich das DL in immer mehr Lehrplänen und PraxisFachzeitschriften einzelner Sportarten.

Dass bewegungswissenschaftlich fundierte Lehrkonzepte ihren Niederschlag in der Sportpraxis finden, begrüßen wir

\footnotetext{
1 Für "differenzielles Lehren“ gibt es keine Definition. Die Vertreter/-innen des DL gehen offenbar davon aus, dass das Anwenden des differenziellen Lernens durch die Lehrperson differenzielles Lehren ist. Zwar ist uns bewusst, dass sich aus Theorien zum Lernen tatsächlich nur bedingt Theorien zum Lehren ableiten lassen; im Folgenden wollen wir uns aber dennoch dem Sprachgebrauch der Vertreter/-innen des DL anschließen, die den Ansatz selbst regelhaft mit "Lehren und Lernen“ überschreiben (z. B. Schöllhorn, 2005; s. auch http://www.sport.unimainz.de/401.php, Zugriff am 22. November 2011). De facto beschäftigen wir uns in diesem Beitrag allerdings - in Entsprechung der vorliegenden Aussagen - allein mit dem differenziellen Lernen.
}

vor dem Hintergrund des Anwendungsbezugs unserer Disziplin ausdrücklich. Die in der Sportpraxis Lehrenden dürfen dabei erwarten, dass wissenschaftlich abgesicherte Erkenntnisse deutlich von Hypothesen und Spekulationen unterschieden werden. Ist dies nicht der Fall, ist es die Aufgabe der Gemeinschaft der Bewegungs- und Trainingswissenschaftlerinnen und -wissenschaftler, auf den hypothetischen Charakter von Hypothesen und den spekulativen Charakter von Spekulationen hinzuweisen. Genau dies soll im Folgenden geschehen. Insbesondere soll verdeutlicht werden, dass es sich beim DL keineswegs um eine wissenschaftlich abgesicherte Methode handelt. Hierzu werden wir nach einer Einordnung des Ansatzes in die bewegungswissenschaftliche Theoriediskussion (1) zeigen, dass die behaupteten Praxiskonsequenzen theoretisch nicht fundiert sind (2), dass die Abgrenzung zu konkurrierenden Lerntheorien lücken- und fehlerhaft ausfällt (3), dass die präsentierte empirische Befundlage auf höchst wackeligen Füßen steht (4) und dass der Ansatz sich auch aus Praxissicht als nicht tragfähig erweist (5). Wir schließen mit einer zusammenfassenden Wertung (6) sowie einer ethischen Reflexion (7) ab.

\section{Bewegungswissenschaftliche Einordnung: Informationsver- arbeitung vs. Selbstorganisation}

In der Bewegungswissenschaft der 1980er Jahre des vorigen Jahrhunderts wurde eine erbitterte Debatte zwischen dem kognitiven Ansatz der Informationsverarbeitung und dem Selbstorganisationsan- satz der dynamischen Systemtheorie um die adäquate Modellierung motorischer Kontroll- und Lernprozesse geführt (vgl. Meijer \& Roth, 1988). Da sich Schöllhorn (1999) bei der Begründung des DL vom Kognitivismus abgrenzt und sich stattdessen auf den systemdynamischen Ansatz bezieht, ist ein kurzer historischer $\mathrm{Ab}$ riss beider Positionen notwendig.

Im Kognitivismus wird von einer Bewegungskontrolle durch zentral gespeicherte Instanzen ausgegangen, wie Schmidt (1975), einer der Hauptvertreter des Informationsverarbeitungsansatzes in der Motorikforschung, in seiner Schematheorie annimmt, durch generalisierte motorische Programme. Soll eine bestimmte Bewegung, z. B. ein Wurf, ausgeführt werden, wird nach dieser Vorstellung das dazugehörige Motorikprogramm aufgerufen. Die spezifische Variante des allgemeinen "Wurfprogramms" wird in Abhängigkeit von dem Ziel (z. B. Wurf auf ein Ziel in $5 \mathrm{~m}$ Entfernung) sowie den situativen Bedingungen (z. B. Wurf mit einem 200-g-Ball) ausgewählt und das Programm damit in angemessener Weise „parametrisiert", bevor es zur Auslösung der Bewegung an die Muskulatur gesandt wird. Das Lernen der regelhaften Beziehung zwischen verschiedenen Zieldetails, situativen Bedingungen und den hierzu passenden Parametern erfolgt als Abstraktion über die beim bisherigen Programmaufruf gesammelten Einzelerfahrungen hinweg (• Abb. 1a). Da diese Abstraktion bei relativ weit auseinanderliegenden Einzelerfahrungen, also

(c) The authors 2012. This article is published with open access at Springerlink.com. 

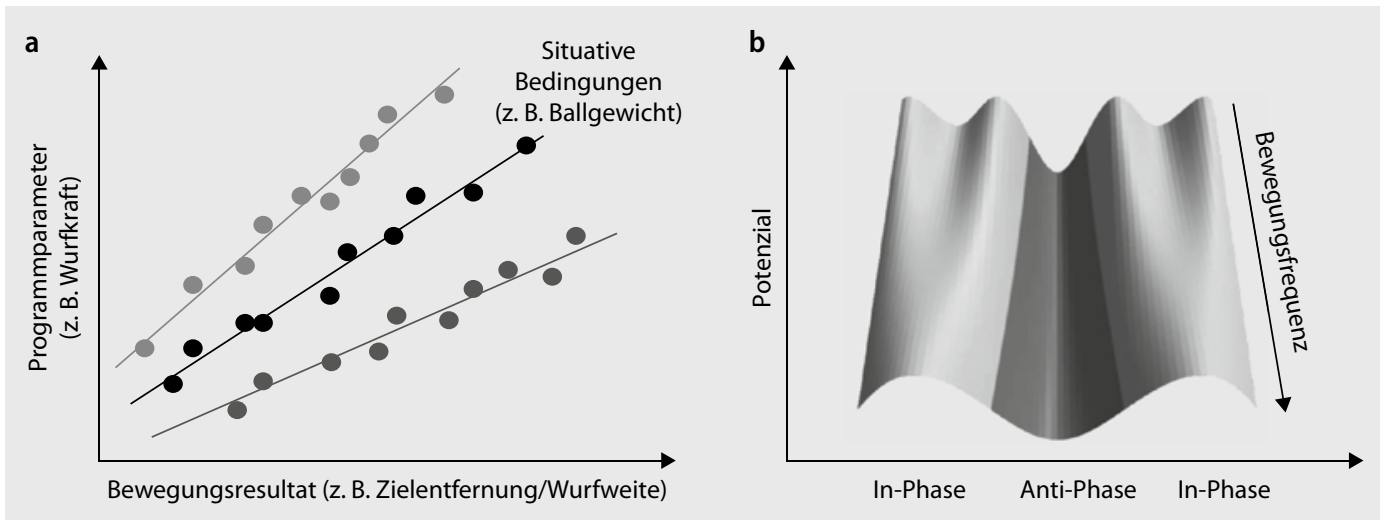

Abb. 14 a Regelabstrak-
tionen für Parametrisierun-
gen eines generalisierten
Motorikprogramms. (Mod.
nach Schmidt, 1975)
b Intrinsische Systemdyna-
mik für gekoppelte Finger-
bewegungen. (Mod. nach
Haken, Kelso \& Bunz, 1985)

z. B. bei Wurferfahrungen auf verschiedene Ziele mit verschiedenen Bällen, exakter ausfällt, ist der Vorteil variablen gegenüber konstanten Übens eine wichtige und vielfach bestätigte Vorhersage der Schematheorie für die Gestaltung des Bewegungsvariationslernens (für eine kritische Übersicht vgl. van Rossum, 1990).

$\mathrm{Zu}$ der Frage nach der optimalen Reihenfolge der Übungsvariation konnte von der kognitiv orientierten Experimentalforschung gezeigt werden, dass eine randomisierte, also eine zufällig verteilte $\mathrm{Ab}$ folge von Bewegungsvariationen im Behaltenstest $\mathrm{zu}$ besseren Leistungen führt als eine geblockte Reihung derselben Variationen (für eine Übersicht vgl. Magill \& Hall, 1990). Offenbar führt die größere Störung (Interferenz) durch die vorangegangenen und nachfolgenden Übungen (Kontext) zu überlegenen Behaltensleistungen. Dieses als Kontextinterferenzeffekt bezeichnete empirische Phänomen wird im Kognitivismus zum einen durch die Elaborationshypothese (Shea \& Morgan, 1979) erklärt, nach der bei einer zufälligen Abfolge sich mehrere Bewegungspläne gleichzeitig im Kurzzeitgedächtnis befinden und daher Gemeinsamkeiten und Unterschiede leichter zu identifizieren sind. In der Rekonstruktionshypothese (Lee \& Magill, 1983) wird zum anderen davon ausgegangen, dass der Bewegungsplan bei einer zufälligen Reihung immer wieder aufs Neue rekonstruiert werden muss und es dadurch zu einer vertieften Informationsverarbeitung kommt.

Im systemdynamischen Ansatz hingegen stehen Selbstorganisationsprozesse des motorischen Systems im Mittelpunkt des Interesses. Die hochdimensionale Bewegungsorganisation geschieht dabei durch niedrigdimensionale Ordnungsparameter. Diese Ordnungsparameter „versklaven“ die Komponenten des Systems, so dass es ausreicht, für die Betrachtung des Gesamtsystems die Ordnungsparameter zu untersuchen.

In der Bewegungsforschung wurde die von Haken, Kelso und Bunz (1985) analysierte Dynamik von Fingerbewegungen zum Standardbeispiel für Selbstorganisationsprozesse der Motorik. Im Selbstversuch zu diesem Beispiel hält man die Hände auf Augenhöhe mit dem Handrücken zum Gesicht. Aufgabe ist es nun, die beiden nach oben gestreckten Zeigefinger im Grundgelenk mit gleicher Frequenz nach rechts und links zu bewegen. Dabei gibt es zwei „attraktive“ Koordinationsmuster: In-Phase, bei der sich die Zeigefinger parallel, und Anti-Phase, bei der sie sich symmetrisch zueinander bewegen. Als Resultat der intrinsischen Systemdynamik („intrinsic dynamics“; Zanone \& Kelso, 1992, S. 404) müssen diese beiden Koordinationsmuster nicht gelernt werden. Vielmehr stellen sie sich unmittelbar „von selbst“ ein. Beginnt man nun mit dem Koordinationsmuster InPhase und erhöht langsam die Frequenz der Bewegungen, wird irgendwann und ohne, dass man dies willkürlich steuern müsste, das Muster In-Phase in das Muster Anti-Phase wechseln. Ab einer gewissen Frequenz existiert also nur noch ein einziger „Attraktor“, in den man zwangsläufig „hineinfällt“. Veranschaulicht wird die sich damit ergebende Systemdynamik gerne als Potenziallandschaft, in der der Ordnungsparameter (hier: die relative Phase zwischen den beiden Zeigefingern) gegen sein Potenzial aufgetragen wird (- Abb. 1b). Der Zustand des Sys- tems wird dabei durch eine Kugel auf der Potenziallandschaft symbolisiert. So, wie die Kugel in der Potenziallandschaft zu Tal rollt, strebt das System durch Selbstorganisation auf ein (lokales) Minimum zu, bei dem sich ein stabiler Zustand befindet, in dem die Kugel also liegen bleibt. Änderungen im sog. Kontrollparameter (hier: Bewegungsfrequenz) schlagen sich dann in Änderungen der Potenziallandschaft nieder: Mit steigender Frequenz wird das Minimum für das Anti-Phase-Muster tiefer, während das lokale Minimum für das In-Phase-Muster langsam verschwindet, so dass bei hoher Frequenz nur noch ein einziger stabiler Zustand übrig bleibt und sich der Systemzustand Anti-Phase in Selbstorganisation ergibt.

Wenn nach Selbstorganisationsansätzen das Bewegungssystem immer einem Minimum zustrebt, kann man Lernen in diesem Ansatz als Veränderung der Systemdynamik verstehen, die sich aufgrund spezifischer Umweltanforderungen („,behavioral information") im Laufe des Lernprozesses einstellt (vgl. z. B. Davids, Button \& Bennett, 2008). In der Folge verändert sich die Potenziallandschaft, so dass neue Minima entstehen, die den gelernten Zuständen entsprechen und auf die nach abgeschlossener Lernphase das System selbstorganisiert zustrebt. In den Worten zweier höchst anerkannter Protagonisten des systemdynamischen Ansatzes, Pier-Giorgio Zanone und Scott Kelso (1992, S. 404):

Learning, in the dynamic pattern framework, is the process by which environmental behavioral information defining a pattern to be learned becomes memorized behavioral information. A coordination pattern is learned to the extent that the intrin- 
sic dynamics are modified in the direction of the to-be-learned pattern. Once learning is achieved, the memorized pattern constitutes an attractor of the behavioral pattern dynamics.

Halten wir also zusammenfassend fest: Während im Kognitivismus Bewegungslernen (u. a.) als Erwerb von motorischen Programmen samt Parametrisierungskompetenzen verstanden wird, optimiert durch variable und randomisierte Übungserfahrungen, sind Lernphänomene aus der Sicht der dynamischen Systemtheorie als Veränderungen der bewegungsaufgabenbezogenen Potenziallandschaft zu interpretieren, wobei durch lernrelevante Verhaltensinformation neue Attraktoren entstehen, denen das System nach Landschaftsumformung selbstorganisiert zustrebt.

\section{DL lässt sich nicht aus dem systemdynamischen Ansatz ableiten}

Wolfgang Schöllhorn bezieht sich bei seiner Herleitung des DL explizit auf den systemdynamischen Ansatz (Schöllhorn, 1999). Die optimale Bewegungslösung entspreche dem globalen Minimum in der Potenziallandschaft, und im DL gehe es darum, dieses globale Minimum zu finden. Dabei sei dieses Minimum für jede Person individuell und deshalb nicht dadurch zu erreichen, dass ein Lehrender einen Sollwert von außen vorgebe und den Lernprozess durch Rückmeldungen steuere. Durch Trainervorgaben bestehe vielmehr die Gefahr, dass die Übenden in einem lokalen Minimum stecken blieben:

Je weniger Möglichkeiten angeboten werden, desto größer ist die Gefahr von Stereotypen, die nicht optimale Lösungen darstellen, da sie meist nicht mehr fähig sind, sich veränderten Bedingungen anzupassen, und es sich daher meist um lokale Minima handelt. Durch eine größere Streuung wird die Wahrscheinlichkeit erhöht, das absolute (auf das Individuum abgestimmte) Minimum zu finden. (Schölhorn, 1999, S. 11)

Weiterhin solle die Streuung maximiert und im Extremfall so gestaltet werden, dass keine Übungsvariation jemals wie-

Sportwiss 2012 - 42:83-95 DOI 10.1007/s12662-012-0251-y

Stefan Künzell · Ernst-Joachim Hossner

\section{Differenzielles Lehren und Lernen: eine Kritik}

\section{Zusammenfassung}

Dieser Beitrag beschäftigt sich kritisch mit dem von Wolfgang Schöllhorn seit 1999 propagierten Ansatz des differenziellen Lehrens und Lernens. Nach einer Einordnung in die historische bewegungswissenschaftliche Debatte um den Informationsverarbeitungsansatz und die dynamische Systemtheorie zeigen wir, dass die von Schöllhorn behaupteten Praxiskonsequenzen theoretisch nicht fundiert sind, dass die Abgrenzung zu konkurrierenden Lerntheorien lücken- und fehlerhaft ausfällt, dass die präsentierte empirische Befundlage auf höchst wackeligen Füßen steht und dass der Ansatz sich auch aus Praxissicht als nicht tragfähig erweist. Mit Blick auf fatale Konsequenzen sowohl für die Sportpraxis als auch für die Sportwissenschaft empfehlen wir, in zukünftigen Publikationen zum differenziellen Lernen auf fehlerhafte und theoretisch wie empirisch unbegründete Praxisempfehlungen zu verzichten.

\section{Schlüsselwörter}

Motorisches Lernen · Differenzielles Lernen · Dynamische Systemtheorie . Informationsverarbeitung

Kontextinterferenz

\section{Differential teaching and learning: a critique}

\section{Abstract}

In this article, the differential learning approach introduced in 1999 by Wolfgang Schöllhorn is critically examined. After positioning this concept in relation to the historical motor-action controversy between information processing and dynamical system theory, we put forward the arguments that consequences claimed for sport practice are not theoretically substantiated, that the tentative demarcation from cognitivism fundamentally fails, that the data presented stands on shaky ground and that, from a practice-related perspective, the approach manifests a severe lack of practicability. In consideration of disastrous consequences for sport practice as well as sport science, we suggest for future publications on differential learning to refrain from deficient and theoretically as well as empirically unsubstantiated recommendations for sport practice.

\section{Keywords}

Motor learning · Differential learning · Dynamical system theory $\cdot$ Information processing $\cdot$ Contextual interference derholt und die optimale Lösung „umkreist“" werde. Auf diese Weise werde „der Lösungsraum (...) an den Rändern abgetastet (...), um die Interpolationsfähigkeit zu trainieren" (Schöllhorn, 2005, S. 133), denn über Interpolationen könne am besten auf die Potenziallandschaft innerhalb des eingekreisten Lösungsraums geschlossen werden.

Zusammenfassend wird im DL der Bezug zur dynamischen Systemtheorie also durch die Behauptungen hergestellt, dass (a) Bewegungslernen dem Auffinden des globalen Minimums entspricht und dass dieses Auffinden (b) durch Verzicht auf Rückmeldungen, (c) durch verstärkte Streuungen sowie (d) durch Abtasten des Lösungsraums vor allem an dessen Rändern erleichtert wird. Dass all dies keineswegs aus der dynamischen Systemtheorie abzuleiten ist, soll im Folgenden im Detail gezeigt werden.

$\mathrm{Zu}$ a) Die wohl eklatanteste Fehlinterpretation der dynamischen Systemtheorie liegt in der Annahme, dass sich das Lernen von Bewegungen auf das Auffinden des globalen Minimums bezieht. Wie weiter oben aufgezeigt, wird in der dynamischen Systemtheorie Bewegungslernen vielmehr durch Veränderungen in der Potenziallandschaft abgebildet. Es geht dabei also nicht um die Suche nach bestehenden Attraktoren, sondern um die Schaffung neuer Attraktoren. Das macht für das Erlernen von Bewegungen folgenden Unterschied: Für das Finden eines bestehenden Attraktors muss man sich in dessen Nähe begeben, die intrinsische Dynamik des Systems sorgt dann dafür, dass man „von alleine“, selbstorganisiert in das Zentrum des At- 
traktors gezogen wird. Oder im Bild: Eine (den Systemzustand symbolisierende) Kugel, die in die Nähe eines Tals gebracht wird, rollt „von alleine“ an die tiefste Stelle und verharrt dort. Dabei entspricht die tiefste Stelle dem Attraktor, der die optimale Lösung symbolisiert. Für das Schaffen eines Attraktors muss sich hingegen die Potenziallandschaft verändern. Erneut im Bild: Das neue Tal muss sich erst ausbilden, bevor dann die Kugel dort hineinrollt und stabil verharren kann.

Entgegen der im DL gemachten Behauptung, dass Bewegungslernen mit dem Auffinden des globalen Potenziallandschaftsminimum gleichzusetzen ist, entsprechen sportliche Bewegungsfertigkeiten jedoch nur in den allerseltensten Fällen bereits bestehenden Attraktoren in der intrinsischen Dynamik - würde dadurch doch die sportliche Aufgabenlösung „,von allein“ erreicht und jedwede Lehrmethodik überflüssig werden. Eine Kippe am Hochreck ist aber ganz offensichtlich kein Attraktor in der intrinsischen Dynamik, den es nur zu finden gilt und in dem das System dann stabil verharrt; dieser Attraktor muss vielmehr neu geschaffen werden durch Verhaltensinformationen.

Wie genau eine solche „Kerbe“ am besten in die Potenziallandschaft hineinzuschlagen ist, wird von der dynamischen Systemtheorie selbst nicht behandelt. Folgt man Hauptvertretern des systemdynamischen Ansatzes in ihren Lernstudien zu gekoppelten Fingerbewegungen, scheint aber zumindest die - nicht weiter begründete - Auffassung zu bestehen, dass Modifikationen der Potenziallandschaft am besten dadurch zu erreichen sind, dass das neu zu erlernende Koordinationsmuster unter ständiger Ergebnisrückmeldung in konstanter Wiederholung geübt wird (Kostrubiec, Tallet \& $\mathrm{Za}$ none, 2006; Tallet, Kostrubiec \& Zanone, 2008, 2010; Zanone \& Kelso, 1992; Zanone, Kostrubiec, Albaret \& Temprado, 2010). Zanone und Kelso (1992) beispielsweise gehen in ihrem Experiment wie folgt vor: „The learning procedure entailed five consecutive daily sessions in which the same required pattern was practiced repeatedly, with knowledge of results given after each trial“ (S. 406) - im exakten Gegenteil also zu dem, was nach dem Ansatz des DL für die Optimierung motorischer Lernprozesse gefordert wird.

$\mathrm{Zu} \mathrm{b)} \mathrm{Wir} \mathrm{haben} \mathrm{oben} \mathrm{bereits} \mathrm{allge-}$ mein festgestellt, dass die dynamische Systemtheorie keine Ableitungen für das optimale Treatment zur permanenten Veränderung der Potenziallandschaft erlaubt. Dies trifft - als zweites Ableitungsproblem des DL - insbesondere für Aspekte der Rückmeldungsgabe zu, also der Bewegungskorrektur. Offenbar besteht bei den Vertreter/-innen des DL die Meinung, dass Lernende in ihrer „selbstorganisierten" Suche nach ihrem individuellen globalen Minimum der Potenziallandschaft durch Korrekturhinweise gestört würden. Wie man dies aus der dynamischen Systemtheorie - die eben eine Kontroll- und keine Lerntheorie ist - ableiten könnte, wissen wir nicht. Allerdings wissen wir definitiv, wie man einen Rückmeldeverzicht beim motorischen Lernen nicht begründen kann: Wenn in der dynamischen Systemtheorie sich die Lichtwellen eines Lasers, die Wassermoleküle in einem Topf oder die Neuronen im Gehirn selbst organisieren, bedeutet dieses „selbst“ ein anderes, als wenn Athleten „selbst“ die optimale Bewegung finden sollen.

$\mathrm{Zu} \mathrm{c)} \mathrm{Eine} \mathrm{dritte} \mathrm{Behauptung} \mathrm{des} \mathrm{DL}$ ist, dass sich die Forderung nach verstärkten Streuungen aus dem systemdynamischen Ansatz ableiten ließe. Dies wird damit begründet, dass „durch Verstärken der Schwankungen während des Aneignungs- und Automatisationsprozesses (...) ein Vorgang der Selbstorganisation beim Athleten gezielt ausgelöst werden [soll]" (Schöllhorn 1999, S. 10). Abgesehen davon, dass das Konzept der Selbstorganisation ad absurdum geführt wird, wenn davon ausgegangen wird, dass sie von außen "gezielt ausgelöst“ werden könne, stellt sich die Frage nach der Natur dieser Prozesse. Schöllhorn und Köpsel (2005) verweisen hierzu darauf, dass beim Übergang von einem Bewegungsmuster zu einem anderen Fluktuationen auftreten. Dieses Phänomen ist tatsächlich zu beobachten (vgl. z. B. Kelso, 1995) und aus systemdynamischer Sicht so zu erklären, dass bei einer Verformung der Potenziallandschaft durch Änderung des Kontrollparameters bestehende Attraktoren flacher werden, bevor sie verschwinden und sich das System in einen neuen stabi- len Zustand bewegt ( $\bullet$ Abb. 1b). Schöllhorn und Köpsel (2005, S. 7) folgern aus diesem Phänomen der kritischen Fluktuationen nun, dass ,aus dieser Sicht (...) Schwankungen bzw. ,Bewegungsfehler als notwendige Voraussetzungen für adaptives Verhalten bzw. Lernen betrachtet werden [können].“

Dies aber ist aus zwei Gründen ein Fehlschluss. Zum ersten betrifft das Phänomen der verstärkten Fluktuationen den reversiblen Vorgang der Zustandsänderung auf kleiner Zeitskala; in dem Fingerexperiment bildet sich also bei wieder verringerter Bewegungsfrequenz der In-Phase-Attraktor zurück. Aus diesen reversiblen Änderungen auf kleiner Zeitskala lassen sich aber in keiner Weise Gesetzmäßigkeiten für irreversible Potenziallandschaftsänderungen auf größerer Zeitskala ableiten, also für Lernprozesse. Zum zweiten - und grundlegender - ist es logisch falsch, aus der Beobachtung einer Begleiterscheinung (hier des verstärkten Rauschens kurz vor einer Systemzustandsänderung) auf dessen Eigenschaft als notwendige Voraussetzung zu schließen, würden damit doch Ursache und Wirkung miteinander vertauscht. Tatsächlich ergeben sich die Fluktuationen, weil der bisherige Attraktor weniger ,attraktiv" wird, umgekehrt jedoch bewirkt eine Erhöhung der Fluktuation von außen keineswegs die Destabilisierung eines bestehenden Attraktors - man wird einfach den Sommer nicht dadurch herbeizwingen können, dass man im Winter Schwalben aussetzt.

$\mathrm{Zu} \mathrm{d)} \mathrm{Ein} \mathrm{vierter} \mathrm{und} \mathrm{letzter} \mathrm{Irrtum} \mathrm{bei}$ der Begründung des DL durch den systemdynamischen Ansatz besteht in der Annahme, ein hochdimensionaler Lösungsraum ließe sich durch Abtasten seiner Ränder „einkreisen“. Dieses Einkreisen ist nach Schöllhorn (1999, S. 9) notwendig, weil künstliche neuronale Netzwerke (die sich in der Tat auch als dynamische Systeme verstehen lassen) recht gut intrapolieren, aber recht schlecht extrapolieren können. Dieses Verhalten extrapoliert Schöllhorn (1999) auf die menschliche Bewegungsorganisation. Das erste Problem dabei ist, dass diese Übertragung von künstlichen neuronalen Netzwerken auf biologische Organismen nicht zwingend ist und in natürlichen neurona- 
len Netzwerken natürlich ganz andere Regeln gelten könnten. Das zweite, konzeptionelle Problem besteht darin, dass das geforderte „Einkreisen“ in mehrdimensionalen Räumen nicht trivial ist: Will man beispielsweise einen zweidimensionalen Lösungsraum durch so eine einfache geometrische Figur wie ein Quadrat begrenzen, dann scheint die Annahme plausibel, dass man mindestens vier Punkte braucht, einen auf jeder Kante des Quadrats, um ein Minimum an „Einkreisen“ zu gewährleisten. In einem dreidimensionalen Würfel sind es dann aber schon acht und bei $n$ Dimensionen $n \times 2^{n-1}$ Kanten. Die Anzahl der abzutastenden „Einkreisungspunkte“ wächst also exponentiell. Schon bei zehn Variationsquellen einer sportlichen Fertigkeit - auf die man bspw. beim Volleyball-Angriffsschlag im Hinblick auf Zuspielhöhe, -weite, -entfernung, Anlaufgeschwindigkeit, -richtung, -rhythmus usw. spielerisch kommt - würden damit aber über 5000 Kanten des Lösungsraums abzutasten sein, um der Forderung nach dessen „Einkreisung" gerecht zu werden, bei 20 Variationsquellen über 10 Mio., was bei täglichem Üben von etwa 500 Variationen eine Übungszeit von über 50 Jahren bedeutete. Dass sich die Forderung nach einem Einkreisen des Lösungsraums an dessen Rändern allein bei Wolfgang Schöllhorn und bei keinen weiteren Vertreter/-innen des systemdynamischen Ansatzes findet, sollte vor dem Hintergrund dieser Zahlen nicht erstaunen.

\section{Die Abgrenzung des DL zum Kognitivismus ist lücken- und fehlerhaft}

Wenn es in der Einleitung zu dem von Schöllhorn und Paschke (2007, S. 28) verfassten Beitrag zum DL im Volleyball lautet: „Gibt es ein neues Zauberwort in der Vermittlung unseres Sports? Wolfgang Schöllhorn und Matthias Paschke von der Uni Münster beleuchten die Hintergründe einer Methode, die die traditionelle Lehrmeinung revolutioniert", dann verweist dies auf zweierlei: auf das angeblich begründete Aufkommen von etwas Neuem und auf die Ablösung von etwas angeblich überholt Altem. Dass es mit der theoretischen Begründung für die ,revolutionär" neue Trainingsmethode nicht so weit her ist, haben wir im vorangegangenen Abschnitt gezeigt. Wenden wir uns von daher im Folgenden dem Alten zu, das angeblich nach Ablösung ruft. In den Aufsätzen zum DL wird dieses Alte im Kern wie folgt beschrieben:

Schaut man sich die übliche Trainingspraxis in Schulen, Vereinen etc. an, so stellt man fest, dass ihr zumeist programmorientierte Lernmodelle zu Grunde liegen (...). In der Konsequenz wird daraus für das motorische Lernen abgeleitet, dass versucht wird, ein vorher definiertes Ideal durch möglichst häufige Wiederholungen der Zielbewegung einzuschleifen. (Schöllhorn \& Köpsel, 2005, S. 4)

Wir werden im Folgenden nachweisen, dass sich diese Konsequenz nicht im Entferntesten aus dem angeblich ,alten“ Kognitivismus ergibt. Insbesondere verdeutlichen wir, dass aus Programmtheorien weder folgt, dass (a) sich ein Motorikprogramm auf eine zuvor definierte Idealbewegung bezieht, noch, dass (b) Bewegungen ,eingeschliffen" werden sollten. Weitergehend stellen wir klar, dass die Abgrenzungsversuche schon allein deshalb nicht überzeugen, weil ganz zentrale Elemente des Informationsverarbeitungsansatzes lücken- oder fehlerhaft dargestellt oder schlichtweg ,vergessen“ werden, was wir an den Beispielen (c) des Kontextinterferenzparadigmas, (d) der kognitiv inspirierten empirischen Rückmeldungsforschung sowie (e) Arbeiten zur Bewegungsvariabilität aufzeigen. Abschließend begründen wir (f) unseren generellen Eindruck, dass wir die Kenntnisse der Vertreter/-innen des DL zum Kognitivismus im Speziellen und zum motorischen Lernen im Allgemeinen nur bedingt fundiert einschätzen.

$\mathrm{Zu}$ a) Wie oben skizziert, definieren sich generalisierte Motorikprogramme nach Schmidt (1975) über einige Merkmale, die über alle Programmvarianten hinweg invariant bleiben, Merkmale also, die z. B. den Wurf zu einem Wurf machen und ihn von einer Schwebekippe unterscheiden. Führt man in dieser Weise den Wurf mit mehr oder weniger Kraft durch, erhält man verschiedene Wurfvarianten; Merkmale wie die zeitliche Abfolge der Muskelimpulse sowie die Relation der investierten Kräfte sollen hingegen invariant bleiben. Wie man sieht, definieren sich Invarianten eines Motorikprogramms also intraindividuell durch über Programmvarianten hinweg unveränderliche Merkmale. Selbstverständlich müssen sich diese intraindividuellen Invarianten bei interindividueller Betrachtung keineswegs in denselben Verlaufsformen niederschlagen, wie Schöllhorn, Beckmann, Janssen und Michelbrink (2009, S. 37) suggerieren: „Sämtlichen traditionellen Ansätzen gemeinsam ist dabei die Annahme einer personenübergreifenden korrekten Zielbewegung, die durch viele Wiederholungen vom Gesamten oder von Teilen (Invarianten) und durch zahlreiche Fehlerkorrekturen ,eingeschliffen' wird." Nicht nur wird der Begriff der „Invariante“ hier fehlerhaft als „Bewegungsteil“ interpretiert; darüber hinaus wird eben der intraindividuelle Charakter der postulierten Invarianten völlig übersehen, der nur bedingt Rückschlüsse auf die individuellen Verlaufsformen der Bewegung zulässt - und definitiv keinerlei Rückschlüsse auf personenübergreifend korrekte Zielbewegungen. Jeder Athlet steuert also mit seinem individuellen Motorikprogramm seine individuelle Bewegung. Der „Parameter der Individualität" (sic!; Schöllhorn, 1999) wird also bei programmtheoretischer Fundierung nicht mehr oder weniger „vernachlässigt“ als bei systemdynamischer.

$\mathrm{Zu} \mathrm{b)} \mathrm{Die} \mathrm{zweite} \mathrm{Fehlinterpretation}$ der Schematheorie ist der Behauptung zu entnehmen, dass "Schmidts Hypothese variablen Übens (...) nur auf die Festigung eines bestehenden ,generalisierten motorischen Programms' ab[zielt]" (Schöllhorn, 1999, S. 11), dass also „im Sinne der Theorie (...) von Schmidt (...) die variablen Parameter variiert werden sollen, um die Invarianten stabiler zu bekommen" (Schöllhorn, 2005, S. 127). Die Quelle dieser Erkenntnis erschließt sich uns nicht, denn, wie auch Shea und Wulf (2005, S. 86) in ihrer kritischen Bestandaufnahme der Schematheorie feststellen: „How GMPs are learned is not addressed in schema theory." Die Theorie von Schmidt bezieht sich also nur auf den Erwerb motorischer Schemata zur Bewegungsvariation und eben nicht auf den Erwerb und die Optimierung von invarianten Programmmerkmalen. 
Diese Fehlinterpretation ist von besonderer Bedeutung. Zwar kann die Schematheorie auf theoretischer Ebene als Gegenpol zu systemdynamischen Ansätzen gesehen werden, dies bedeutet jedoch nicht, dass sie einem variablen Üben entgegensteht. Ganz im Gegenteil ist es ja der Kern der Schematheorie, dass variables Üben als notwendige Voraussetzung für die Ausbildung zuverlässiger Schemaregeln angesehen wird. In Schmidts Theorie werden lediglich die Grenzen und Konstruktionsregeln für variables Üben - wie wir noch sehen werden: im Gegensatz zum DL - genau definiert. Da sich die Theorie aber zum Erwerb von Programminvarianten neutral verhält, kann man sie nicht als "Schuldige" für die theoretische Begründung von Lehrmethoden heranziehen, bei denen „die ,korrekte ' Lösung mit hohen Wiederholungszahlen eingeschliffen werden [soll] “ (Schöllhorn, 2005, S. 127). Im Gegenteil, in der Schmidt-Gruppe wird ein eventueller Vorteil von Variationen auch über Programmgrenzen hinweg als eine offene, empirische Frage gesehen. Die Schmidt-Schülerin Gabriele Wulf geht in ihrer Habilitationsschrift (1994) genau dieser Frage experimentell nach. Diese Arbeit, erschienen in der HofmannSchriftenreihe der Beiträge zur Lehre und Forschung im Sport, einem deutschsprachigen Sportwissenschaftler also durchaus zugänglich, wird in den gesamten Arbeiten der Vertreter/-innen des DL mit keinem einzigen Wort erwähnt. ${ }^{2}$

Zu c) Schöllhorn (2000, S. 79, Abb. 6; und wiederholt Schöllhorn 2010, S. 15) grenzt den "neuen“ Ansatz des DL von den traditionellen kognitiven Ansätzen dadurch ab, dass im DL Übungsvariationen in zufälliger Abfolge (ACDBA...) darzubieten seien, während die traditionelle Sicht eine blockweise Darbietung (AAAAB...) favorisiere. Dies kann aus motorikwissenschaftlicher Sicht nur verwundern - beruht doch auf genau diesem Unterschied zwischen randomisiertem

\footnotetext{
2 Objektive Grundlage dieser Feststellung sowie weiterer Aussagen zu Publikationen der Vertreter/-innen des DL bilden sämtliche von Wolfgang Schöllhorn auf der Internetseite zum DL für den Zeitraum 1999-2011 zum DL aufgeführten Publikationen (vgl. http://www.sport. uni-mainz.de/401.php, Zugriff am 22. November 2011).
}

und blockweisem Üben, wie oben skizziert, die empirische Überprüfung des Kontextinterferenzparadigmas des Informationsverarbeitungsansatzes. Der Umstand, dass der Kognitivismus mit dem Ansatz der Elaboration oder der vertieften Verarbeitung die viel sparsamere Erklärung für den Vorteil randomisierten Übens liefert, wurde von den Vertreter/innen des DL aber offenbar erst 2005 entdeckt (erstmalige Erwähnung: Schöllhorn, 2005, S. 132).

In der Folge versucht Schöllhorn nachzuweisen, dass sich „differenzielles Lernen (...) in dem Ziel, der Vorgehensweise und den praktischen Konsequenzen vom randomisierten Cl-Lernen [unterscheidet]“ (2005. S. 133; CI für „Context-Interferenz"). Als einen wesentlichen Unterschied identifiziert er, dass sich das Kontextinterferenzlernen auf „mehrere zu erlernende Gegenstände“ bezieht, während DL „auf das Erlernen eines(!) (wenn auch ständig veränderlichen) Gegenstandes ab[zielt]“" (Schöllhorn 2005, S. 133). Dies aber ist für die Begründung eines konzeptuellen Unterschieds irrelevant, denn unabhängig davon, ob man auf die Optimierung eines einzigen Bewegungsmusters oder mehrerer abzielt, ist (für eines oder jedes dieser Muster) entweder eine Übungsabfolge ACDBA... gegenüber einer Übungsabfolge AAAAB... von Vorteil oder eben nicht. Der positive Fall ist als Bewährung des Kontextinterferenzansatzes zu interpretieren und - wenn man sich der DL-Herleitung dieser Vorhersage anschließen mag - zugleich als Bewährung des DL-Ansatzes, der negative Fall als Belastung beider Ansätze. Eine theoretisch fundierte Abgrenzung vom Kontextinterferenzansatz bleiben die Vertreter/-innen des DL damit schuldig (zum Aspekt der auf die empirischen Lernkurven bezogenen „praktischen Konsequenzen“s. 4b).

$\mathrm{Zu} \mathrm{d)} \mathrm{In} \mathrm{die} \mathrm{im} \mathrm{Rahmen} \mathrm{des} \mathrm{DL-An-}$ satzes vorgelegten empirischen Gruppenvergleiche fließen auch immer wieder Aspekte der Bewegungskorrektur ein, wobei sich das differenzielle Training dadurch auszeichnet, dass es ,in Anlehnung an (Schöllhorn, 1999) und (Römer et al., 2003) ohne Wiederholungen und Fehlerkorrekturen [durchgeführt wird] “ (Schöllhorn, Paschke \& Beckmann, 2006, S. 102), während im klassischen Training „die Bewegungsrealisation durch häufige Korrekturhinweise eng an die erwünschte Zielbewegung angeglichen [wird]" (Römer, Schöllhorn, Jaitner \& Preiss, 2003, S. 131). Überraschenderweise werden diese Aussagen zur optimalen Rückmeldefrequenz beim Bewegungslernen getroffen, ohne dass jemals auch nur auf einen einzigen Befund aus der Vielzahl der hierzu vorliegenden empirischen Resultate Bezug genommen würde, die dem kognitiv inspirierten Forschungsprogramm zur „Feedback-Vermehrung“ entsprungen sind. In amerikanischen Lehrbüchern sind hier typischerweise ganze Kapitel dieser Thematik gewidmet - in der zum Zeitpunkt der ersten Publikationen zum DL verfügbaren Auflage des Magill-Lehrbuchs (1998) sind es über 50 Seiten!

Es hätte allerdings auch schon ein Blick in den von Marschall und Daugs (2003) im Handbuch Bewegungswissenschaft/Bewegungslehre publizierten Überblicksbeitrag ausgereicht, um zu lesen, dass erstens die Forderung nach völligem Verzicht auf ergänzende Rückmeldungen zur Ausführungsgüte schlichtweg falsch ist und der Lernfortschritt dadurch definitiv behindert wird, dass dies zweitens aber nicht bedeutet, dass zu jeder Bewegungsausführung ergänzende Rückmeldungen gegeben werden sollten, denn reduzierte Rückmeldehäufigkeiten sind bekanntermaßen von Vorteil (vgl. z. B. Winstein \& Schmidt, 1990). Im Unterschied zum DL bietet der Informationsverarbeitungsansatz zum Vorteil reduzierter Rückmeldehäufigkeiten mit der Guidance-Hypothese (Schmidt, 1991) eine einfache und sparsame Erklärung an: Rückmeldungsgabe bei jeder Bewegungswiederholung macht die Lernenden von der extern gegeben Information „abhängig“; im Lernprozess geht es aber darum, dass sie sich einen eigenen, internen Referenzwert für die korrekte Bewegungsausführung aneignen. Da die Lernenden in Versuchen ohne externe Rückmeldung selbst die Bewegungsgüte hinterfragen müssen, wird diese Aneignung durch reduzierte Rückmeldefrequenzen gefördert. Welche theoretischen Begründungen gibt uns im Vergleich hier$\mathrm{zu}$ das DL? Und warum wird in sämtlichen Publikationen der Vertreter/ -innen des DL auf keinen einzigen empirischen 
Befund aus der Rückmeldungsforschung Bezug genommen?

$\mathrm{Zu}$ e) Nach all dem bisher Gesagten mag es kaum mehr erstaunen, dass im DL nicht nur, wie oben geschildert, die Schematheorie in ihren Aussagen zur Stabilität und Variabilität im Bewegungsverhalten fehlerhaft referiert wird, sondern dass auch solche Arbeiten zur Bewegungsvariabilität mangelhaft wiedergegeben werden, die über das Konzept der motorischen Programme hinausgehen. So findet sich beispielsweise bei Schöllhorn (2005, S. 127) die mit explizitem Verweis auf Müller (2001) versehene Behauptung, dass „jüngere Forschungsarbeiten [jenseits des DL] zum Thema der Variabilität und Stabilität von Bewegung (...) die Nichtwiederholbarkeit nur implizit zum Gegenstand [haben und dass] (...) demnach Variabilität in einer Bewegungsausführung als ein nicht zu vermeidendes ,Übel' in Kauf genommen [wird]“. Diese Behauptung lässt sich wohl allein so erklären, dass die zitierte Arbeit nicht im Original studiert wurde, denn Müller (2001) unterscheidet doch ganz ausdrücklich Rauschanteile an der Bewegungsvarianz von solchen Anteilen, die von der Person zur Steigerung der Ergebniskonstanz genutzt werden können. Wenn über diese bloße Beschreibung hinaus noch ein Ansatz zur algorithmisch exakten Zerlegung verringerter Bewegungsvarianz im Laufe des Lernprozesses in Anteile der Rauschreduktion, der Stabilität sowie der Kovariation angeboten wird, dann handelt es sich bei der Arbeit von Müller (2001) um einen Beitrag zur Bewegungswissenschaft, der in seinem theoretischen Gehalt ganz offenbar weit über die schlichte - und, wie im Folgenden deutlich wird: nicht einmal empirisch fundierte - Behauptung des DL-Ansatzes hinausgeht, dass Rauschen grundsätzlich etwas Positives darstellt.

$\mathrm{Zu} \mathrm{f)} \mathrm{Bis} \mathrm{hierhin} \mathrm{kann} \mathrm{festgehalten}$ werden, dass die von den Vertreter/-innen des DL vorgenommene Abgrenzung zum klassischen Ansatz des Kognitivismus auf Fehlinterpretationen sowie einer weitgehenden Ignoranz der im Rahmen dieses Ansatzes vorgelegten Arbeiten beruht. Die Oberflächlichkeit des diesbezüglichen Wissens verdeutlicht sich an vielen Stellen, z. B. wenn „programmorien- tierte Lernmodelle“ (Schöllhorn \& Köpsel, 2005, S. 4) diskutiert werden (es handelt sich um Theorien zur motorischen Kontrolle), wenn die Schematheorie „als eine Form der Closed-loop-Modelle“ bezeichnet wird (Schöllhorn, 2005, S. 126; sie bezieht explizit open-loop-kontrollierte Bewegungen mit ein) oder wenn „programmorientierte[n] Bewegungsmodelle[n] " wie der Schematheorie ein konzeptionelles Speicher- und Neuigkeitsproblem unterstellt wird (Schöllhorn \& Köpsel, 2005, S. 5) (genau dies wird in der Schematheorie thematisiert und gelöst).

Erschwerend kommt hinzu, dass 1999, dem Jahr der ersten Veröffentlichung zum DL (Schöllhorn, 1999), auch innerhalb des Informationsverarbeitungsansatzes die Uhr für die Schematheorie von Schmidt (1975) längst abgelaufen war. $\mathrm{Zu}$ diesem Punkt hätte beispielsweise in dem von Roth und Willimczik vorgelegten Lehrbuch zur Bewegungswissenschaft (1999, S. 194-199, S. 207) nachgelesen werden können, dass es innerhalb des Informationsverarbeitungsansatzes auch alternative Programmkonzepte gibt, die Freiraum für Prozesse der peripheren Selbstorganisation lassen (z. B. Rosenbaum, Kenny \& Derr, 1983), und dass zudem seit Beginn der 1990er Jahre die Idee einer effektkontrollierten Motorik eine zunehmende Bedeutung erfahren hatte, in der deutschsprachigen Sportwissenschaft vor allem in Form des Modells der antizipativen Verhaltenskontrolle von Hoffmann (1993). ${ }^{3}$ Da sich solche Modelle auf die Erreichung intendierter Zielzustände beschränken, den Weg zu diesen Zuständen aber offen lassen und auf diese Weise - zumindest implizit - Prozesse der peripheren Selbstorganisation mitdenken, konnte in den 1990er Jahren eine deutliche Annäherung

\footnotetext{
3 Aus Platzgründen können wir diesen Gedanken hier nicht ausführen und verweisen daher darauf, dass wir dies an anderer Stelle unter dem Gegensatzpaar der Präskription vs. Emergenz getan haben (Hossner \& Künzell, 2003). Die Schematheorie von Schmidt (1975) wird dort als prototypisches Beispiel für eine präskriptive Theorie diskutiert, da „selbst die kleinsten Details der Bewegung zentral (...) kontrolliert werden. In dieser weitreichenden Art, vorschreibende', präskriptive Theorien werden [aber] seit Beginn der 90er Jahre des 20. Jahrhunderts in der Bewegungswissenschaft nicht mehr ernsthaft diskutiert" (S. 137).
}

zwischen den Positionen der Informationsverarbeitung und der dynamischen Systemtheorie verzeichnet werden, die spätestens mit der Publikation von Pieck (1998) in eine weitestgehende Auflösung des in den 1980er Jahren noch schwelenden Konflikts der Motor-action-Kontroverse (Meijer \& Roth, 1988) mündete.

Es hat sich also einiges getan in der Motorikwissenschaft der letzten 25 Jahre. Wenn aber Schöllhorn noch 2005 als Urheber "grundlegende[r] Bewegungsmodelle“ zur Begründung „traditioneller Formen des Lehrens und Lernens“ allein Lashley (1917), Adams (1971) und Schmidt (1975) aufzuzählen weiß (Schöllhorn, 2005, S. 125, 126) und diese "grundlegend andere[n] Zugängen“ gegenüberstellt, z. B. den „Arbeiten zur Selbstorganisation (Haken, Kelso \& Bunz, 1985; Schöner \& Kelso, 1988)“, dann hat er sämtliche Entwicklungen der jüngeren Vergangenheit vollständig verpasst.

\section{Der Nachweis der Wirksamkeit des DL wird empirisch nicht erbracht}

Nun könnte es aber sein, dass trotz fehlerhafter und mangelnder theoretischer Begründung die abgeleiteten DL-Methoden dennoch von Relevanz wären, denn aus Falschem folgt bekanntlich Beliebiges. Es liegt daher nahe, die von den Vertreter/-innen des DL selbst vorgelegten Experimente etwas genauer unter die Lupe zu nehmen. Im Mittelpunkt dieser Studien steht regelhaft der Vergleich zweier Versuchsgruppen, von denen während einer Treatmentphase eine nach dem DL trainiert, während die andere ein als „traditionell“ oder „klassisch“ bezeichnetes Übungsprogramm durchführt. Verglichen werden dann die Leistungen der beiden Gruppen direkt nach Abschluss der Treatmentphase in einem Posttest, ggf. ergänzt im Hinblick auf vergleichbare Ausgangsniveaus durch einen Pretest oder auch im Hinblick auf überdauernde Lerneffekte durch einen oder mehrere Retentionstests (durchgeführt also nach einer längeren Pause nach Ende der Treatmentphase).

Gegen diesen grundlegenden Versuchsplan ist zunächst nichts einzuwenden; nichtsdestoweniger wollen wir im 
Folgenden einige wichtige Details (a) zum experimentellen Design, (b) zur Interpretation empirischer Daten, (c) zu Verfahren der statistischen Auswertung und (d) zur Publikation erhaltener Resultate thematisieren. In all diesen Aspekten werden wir erhebliche Schwächen aufdecken, die zusammenfassend in den Schluss münden, dass von den Vertreter/-innen des DL keine ernst zu nehmende empirische Evidenz für die angebliche Überlegenheit des DL vorgelegt wird.

$\mathrm{Zu}$ a) Verdeutlichen wir die Logik experimentellen Forschens zunächst an einem nichtsportlichen Beispiel. Wenn ein Waschmittelhersteller überprüfen will, ob die Zugabe eines Wirkstoffs W zu besseren Waschergebnissen führt, wird er sinnvollerweise die Waschergebnisse vergleichen, die seine bisherige Mischung im Vergleich zur Mischung mit W-Zusatz erbringt. In diesem experimentell-kontrollierten Vergleich wird er tunlichst alle anderen Faktoren, die das Waschergebnis beeinflussen könnten, konstant halten, also z. B. mit derselben Waschmaschine mit demselben Programm vergleichbar verschmutzte Wäsche waschen. Ist das Ergebnis nach $\mathrm{W}$-Zusatz besser, kann man den Unterschied eindeutig auf die Hinzufügung zurückführen.

Die hiermit skizzierte Grundregel zur Erstellung experimenteller Designs missachten die Vertreter/-innen des DL in jedem der von ihnen präsentierten Experimente, indem die im DL behaupteten Mechanismen eben gerade nicht der Prüfung unterzogen werden und stattdessen nur globale Gruppenvergleiche erfolgen. Wenn aber die DL-Hypothese getestet werden soll, dass Differenzen zwischen aufeinander folgenden Wiederholungen maximiert werden sollen, dann muss man eine solche Gruppe mit einer anderen vergleichen, in der dieselben Variationen in einer Abfolge dargeboten werden, die zu geringeren mittleren Differenzen führt. Und wenn die DL-Hypothese getestet werden soll, dass Rückmeldungen den Lernerfolg behindern, dann muss man eben eine Gruppe ohne Rückmeldungen mit einer anderen vergleichen, in der bei ansonsten identischem Übungsprogramm Rückmeldungen gegeben werden. Verändert man das Treatment hingegen in mehrfacher Hinsicht - im obigen
Bild: nimmt man zusätzlich zum Waschmittelzusatz noch eine andere Waschmaschine -, dann weiß man eben nicht, ob aufgedeckte Unterschiede auf den Zusatz, auf die andere Maschine oder auf beides zurückzuführen sind. Wenn man dies aber nicht weiß, darf man die Befunde auch nicht auf den Waschmittelzusatz zurückführen. Mit anderen Worten: Keines der vorgelegten Experimente lässt sich als empirische Untermauerung der behaupteten Mechanismen interpretieren, die dem DL zugrunde liegen sollen.

Erschwerend kommt hinzu, dass das Treatment der „traditionellen“ Vergleichsgruppe in den vorgelegten Experimenten ungeschickterweise so gewählt wird, dass für dieses Treatment manchmal auch die Schematheorie, in jedem Fall aber der Kontextinterferenzansatz geringere Lernleistungen der Vergleichsgruppe vorhersagen würde. Wenn aber sämtliche Erklärungsansätze Vorteile derselben Versuchsgruppe vorhersagen, dann kann man aus der empirischen Bestätigung dieser Vorhersage nicht folgern, dass sich das DL als den anderen Erklärungen überlegen erweist. In jeder der von den Vertreter/-innen des DL vorgelegten Studien wird nicht mehr geboten als genau dies! Um im obigen Bild zu bleiben: Gezeigt wird lediglich, dass Kernseife schlechtere Waschergebnisse bringt als das „neue“ Waschmittel. Ob dieser schlichte Befund Anhänger/-innen „herkömmlicher“ Waschmittel überzeugen kann?

$\mathrm{Zu}$ b) Denken wir den aktuellen Gedanken noch ein wenig weiter, dann kommt man zu der Frage, wie denn ein angemessener Vergleich von differenziellem und kontextinterferenzbasiertem Lernen aussehen könnte. Kann man sich ein Treatment vorstellen, bei dem nach dem DL von Versuch zu Versuch erheblich variiert wird, ohne dass zugleich nach dem Kontextinterferenzansatz der Bewegungsplan von Versuch zu Versuch rekonstruiert werden müsste? Wohl kaum. Die empirischen Ergebnisse, also Lernkurven und Gruppendifferenzen, sollten aus diesem Grunde bei Experimenten zum DL nicht anders ausfallen, als wenn dieselben Experimente der Überprüfung des Kontextinterferenzeffekts gedient hätten.

Ganz grundsätzlich ist an dieser Stelle festzuhalten, dass auf empirischer Ebene gar nicht sichtbar werden kann, ob Experimente auf theoretischer Ebene mit dem Ziel der Überprüfung der Rauschverstärkungshypothese des DL oder der Rekonstruktionshypothese des Kontextinterferenzansatzes entworfen wurden. Schöllhorn (2005, S. 133) scheint aber genau hiervon auszugehen und tatsächlich anzunehmen, dass die von den Vertreter/-innen des DL durchgeführten Experimente einen anderen Verlauf genommen hätten, wenn sie im Rahmen der Kontextinterferenzforschung durchgeführt worden wären:

Die Charakteristiken der Lernkurven (...) legen nahe, dass es sich beim differenziellen Lernen um ein anderes Phänomen handelt als im Cl-Lernen [dem Kontextinterferenz-Lernen]. Im Falle des differenziellen Lernens sind die Lernfortschritte schon am Ende der Intervention deutlich im Vorteil gegenüber anderen Ansätzen und werden in den Retentionstest noch größer. In den zu Cl-Experimenten vergleichbaren Zeitintervallen ist beim differenziellen Lernen kein Rückgang wie in den meisten anderen Lernansätzen zu beobachten, sondern stattdessen ein Ansteigen der Leistung.

Im Klartext: Würde beispielsweise Ballmaterial in einer DL-Studie variiert werden, erwarteten die Vertreter/-innen des DL eine hohe Stabilität des Lernresultats; würde hingegen Ballmaterial in derselben Weise in einer KontextinterferenzStudie variiert werden, sagten sie einen Leistungsrückgang vorher - was für ein unsäglicher Unfug! In den empirischen Wissenschaften wird - mit gutem Grund - von einer Unabhängigkeit der empirischen Beobachtung von der zu prüfenden theoriebezogenen Hypothese ausgegangen. Wir wollen deshalb den vorgebrachten Abgrenzungsversuch zwischen DL und Kontextinterferenzeffekt nicht ernst nehmen.

$\mathrm{Zu} \mathrm{c)} \mathrm{Ein} \mathrm{wichtiger} \mathrm{Grundsatz} \mathrm{empi-}$ rischer Forschung besteht darin, die eigenen Befunde der Überprüfung durch die wissenschaftliche Gemeinschaft zugänglich zu machen. Empirische Beiträge sind von daher üblicherweise so aufgebaut, dass eine ausführliche Beschreibung der verwendeten Methoden und Verfahren erfolgt und die erhaltenen Resultate so wiedergegeben werden, dass sich den Le- 
ser/-innen die Bedeutsamkeit wie die Verallgemeinerbarkeit der präsentierten Befunde nachvollziehbar erschließt.

Ein Positivbeispiel der zum DL vorliegenden Studien bildet das Experiment zum Start im Eisschnelllauf (Savelsbergh, Kamper, Rabius, De Koning \& Schöllhorn, 2010). In diesem Beitrag findet man detaillierte Beschreibungen der Treatments, eindeutige Berechnungsangaben $\mathrm{zu}$ den verwendeten Variablen sowie eine varianzanalytische Überprüfung der erzielten Pre- und Posttest-Zeiten auf Unterschiede samt Post-hoc-Tests auf Detailursachen dieser Unterschiede. Diese Angaben erlauben es ohne größere Probleme, den Versuch einer Replikation der Studie in Angriff zu nehmen. ${ }^{4}$

In der deutlichen Mehrzahl der sonstigen empirischen Beiträge zum DL ist dies nicht der Fall. Hier mag es in Aufsätzen in Praxiszeitschriften noch akzeptabel sein, wenn die Ergebnisse der DL-Studien zusammenfassend als „signifikant“ bezeichnet werden, ohne dass das Rechenverfahren oder die zu Grunde liegenden statistischen Werte publiziert würden (z. B. Schöllhorn \& Paschke, 2007). Allerdings ist auch für wissenschaftliche Publikationen festzustellen, dass das Rechenverfahren mitunter gar nicht erst angegeben wird (z. B. Schöllhorn, Beckmann, Janssen \& Drepper, 2010) oder die statistischen Parameter fehlen, die eine genaue Einordnung des Befunds erlauben würden (z. B. Beckmann \& Schöllhorn, 2006). Keine einzige Studie zum DL über die oben genannte hinaus haben wir gefunden, in denen A-priori-Kalkulationen von optimalen Stichprobenumfängen vorgenommen werden, eine Vorab-Diskussion und -Festlegung des kritischen Niveaus für die Signifikanzprüfung erfolgt, varianzanalytisch an die Überprüfung der zentralen Interaktionsvorhersagen herangegangen oder eine Begründung für ein alternati-

\footnotetext{
${ }^{4}$ Da es an dieser Stelle um Darstellungsaspekte geht und weniger um die dargestellten Resultate selbst, sei nur am Rande angemerkt, dass sich in dieser Studie - obwohl die traditionelle Gruppe konstante Übungserfahrungen sammelte (vgl. hierzu 4a) - keine signifikanten Unterschiede zwischen differenzieller und traditioneller Gruppe ergaben:, „There were no significant differences between the traditional and differential groups" (Savelsbergh et al., 2010, S. 423).
}

ves Verfahren gegeben wird oder erhaltene Effektgrößen berechnet und interpretativ eingeordnet werden.

Über diese Verstöße gegen empirische Forschungsstandards hinaus wiegt es im Hinblick auf die Nachvollziehbarkeit des experimentellen Designs noch schwerer, dass durchgängig auch auf eine exakte Beschreibung der Gruppentreatments (unabhängige Variablen) und der Berechnungsvorschriften für die Testwerte (abhängige Variablen) verzichtet wird. So finden sich beispielsweise in der Originalveröffentlichung der von Trockel und Schöllhorn (2003; vgl. auch Schöllhorn et al., 2004) durchgeführten Torschussstudie zwar allgemeine Hinweise zur experimentellen Gestaltung des differenziellen Trainings, nämlich, dass Variationen im Hinblick auf Standbein, Schussbein, Körpergeometrie, Oberkörper, Armhaltung und Ball durchgeführt wurden (Trockel \& Schöllhorn, 2003, S. 104-105); Angaben dazu, wie diese Variationen genau aussahen, wie sie gereiht wurden und wie viele Variationsversuche pro Trainingseinheit $\mathrm{zu}$ absolvieren waren, fehlen aber vollständig. Was zudem in dieser beispielhaften Studie die Angaben zur Aggregation des Testwerts angeht: Hier muss man sich schon die Original-Staatsexamensarbeit von Trockel (2002) besorgen, um zu erfahren, dass in Pre- und Posttest die Zielgenauigkeitsleistung des Torschützen mit einem aktiven Torhüter erhoben wurde - ein im Hinblick auf die Durchführungsobjektivität der Studie aus unserer Sicht doch recht relevanter Aspekt.

Zusammenfassend ist festzuhalten, dass die deutlich überwiegende Mehrzahl der zum DL vorliegenden Experimente in einer Weise publiziert vorliegt, die gängigen Standards der empirischen Forschung nicht genügt. Weder erlauben die Darstellungen das eindeutige Nachvollziehen der statistischen Resultate noch die Planung von Replikationsstudien. Dies aber bedeutet, dass es eine Frage des Glaubens wird, ob man den Autor/-innen in ihren Schlussfolgerungen folgen will oder nicht - mit wissenschaftlicher Forschung hat dies wenig zu tun.

$\mathrm{Zu}$ d) In einer im Internet veröffentlichten „Gegendarstellung“ reagieren Schöllhorn, Beckmann und Janssen (2009) auf einen von Wolfgang Killing (2009), dem
Leiter der Mainzer DLV-Trainerschule, in der Zeitschrift leichtathletiktraining publizierten Beitrag, in dem sich dieser kritisch mit dem Ansatz des DL auseinandersetzt. Insbesondere wird dabei Killings Vorwurf, dass die von den Vertreter/-innen des DL vorgelegten Befunde vor allem auf Testleitereffekte zurückzuführen seien, zu entkräften versucht. Hiergegen argumentieren die Kritisierten,

dass der bislang größte Teil der Studien zum Differenziellen Lernen von den Untersuchungsleitern eher mit der Intention des Widerlegens durchgeführt wurde. D. h. bei eventuellem Vorliegen eines Versuchsleitereffektes würden die Ergebnisse eher eine Unter-als einer Überschätzung aufweisen. (Schöllhorn, Beckmann \& Janssen, 2009, S. 3)

Dieser Vorsatz des absichtsvollen Widerlegens des eigenen Ansatzes ist über jeden ethisch-moralischen Zweifel erhaben; es erstaunt vor dem Hintergrund dieser Aussage nur der tatsächliche Umgang mit in genau diesem Sinne „erfolgreichen“ Versuchen. So findet man im Abstract-Band zur Jahrestagung der Arbeitsgemeinschaft für Sportpsychologie 2006 in Münster den Bericht über ein Experiment von Rehers, Beckmann und Schöllhorn (2006), in dem eine differenzielle Gruppe im Vergleich zu einer traditionellen Gruppe nach acht Trainingseinheiten zum Dribbeln im Fußball nicht nur keine signifikanten Unterschiede in der Verbesserung vom Pre- zum Retentionstest, sondern in der Tendenz sogar die geringeren Lernzuwächse verzeichnet. Wenn also tatsächlich „der bislang größte Teil der Studien (...) eher mit der Intention des Widerlegens durchgeführt wurde“: Warum wird diese Studie zum DL in keiner späteren Zusammenfassung der Befundlage erwähnt? Und warum findet man die Studie nicht einmal auf der von Wolfgang Schöllhorn und Mitarbeiter/-innen gepflegten Internetseite (http://www.sport.uni-mainz. de/401.php, Zugriff am 22. November 2011), auf der man eine Vielzahl von $\mathrm{Pu}$ blikationen, einschließlich einseitiger Tagungs-Abstracts, zum DL aufgelistet findet? Sollte hier vielleicht doch eine schönende Auswahl getroffen worden sein die dann den Hinweis auf die „Intention des Widerlegens“ als Entgegnung des Vor- 
wurfs von Testleitereffekten deutlich relativieren ließe? ${ }^{5}$

\section{Der Sportpraxis liefert das DL nichts Neues}

Für die Trainingslehre unterscheiden Hohmann, Lames und Letzelter (2007) wissenschaftlich bewährte Wissensbestände (die aus diesem Grunde der Trainingswissenschaft zugerechnet werden) von solchen Wissensbeständen, die zwar nicht wissenschaftlich bewährt sind, nichtsdestoweniger aber als „Erfahrungswissen " die Trainingspraxis bereichern. In Übertragung dieses Ansatzes auf die Bewegungswissenschaft muss man nach all dem Gesagten feststellen, dass DL definitiv nicht als bewegungswissenschaftliches Konzept bezeichnet werden kann. Dies schließt aber nicht aus, dass sich die gegebenen Empfehlungen trotz fehlender Begründung dennoch für die Sportpraxis als hilfreich erweisen könnten. Dass tatsächlich nicht einmal dies der Fall ist, wollen wir im Folgenden aufzeigen. Im Detail werden wir dabei nachweisen, dass (a) die aus Praxisperspektive gegebenen Begründungen für das DL jeder Logik entbehren, dass (b) die „traditionelle“ Lehrweise verzerrt dargestellt wird und dass (c) der Ansatz es grundlegend an Praktikabilität vermissen lässt.

$\mathrm{Zu}$ a) Jenseits der bereits diskutierten theoriebezogenen Begründungsversuche für das DL findet man in Publikationen der Vertreter/-innen des DL immer wieder das Argument der Nichtwiederholbarkeit identischer Bewegungen, bspw. in der Formulierung von Schöllhorn et al. (2004, S. 13): „Tritt eine Bewegung jedoch kein zweites Mal in identischer Form auf, wird das Ziel des Einschleifens von Bewegungsabläufen (...) grundlegend in Frage gestellt." Die Logik dieser Aussage er-

\footnotetext{
5 Da dies keinen spezifisch DL-bezogenen inhaltlichen Punkt ausmacht, verzichten wir hier auf die ausführliche Dokumentation weiterer Überraschungen im Hinblick auf die von Vertreter/-innen des DL gezeigte Publikationskultur. Geneigten Leserinnen und Lesern empfehlen wir aber in diesem Zusammenhang das genaue vergleichende Studium der Beiträge von Schöllhorn (1999) und Schöllhorn (2000) und insbesondere auch von Schöllhorn (2005, S. 130-133) und Schöllhorn (2010, S. 15-19).
}

schließt sich uns nicht. Wird hier wirklich behauptet, dass sich aus dem Umstand, dass etwas nicht perfekt erreicht werden kann (hier: tatsächliche Bewegungskonstanz), ableiten lässt, dass man dies gar nicht erst versuchen sollte (hier: intendierte Bewegungskonstanz)? Würde man also - in entsprechender Weise - aufgrund des Faktums, dass es einem erwachsenen Deutschen kaum gelingen kann, ein akzentfreies Englisch als Zweitsprache zu erwerben, dieses anspruchsvolle Ziel ,grundlegend in Frage stellen“ wollen, oder aus der höchst geringen Wahrscheinlichkeit, nie wieder zu erkranken, wirklich folgern wollen, dass man es mit Krankheitsprävention gar nicht erst versuchen sollte?

$\mathrm{Zu} \mathrm{b})$ Seit der Erstveröffentlichung zum DL, in der Schöllhorn (1999) nach dem ,vernachlässigten Parameter Individualität" fragt, wird wiederholt behauptet, dass nicht nur aufgrund veralteter kognitiver Fundierung, sondern auch aus der praktischen „Meisterlehre“ heraus in der traditionellen Techniktrainingsmethodik das „Einschleifen“ von Bewegungstechniken favorisiert werde: „Dabei sollen ,Fehler' vermieden werden und (...) die ,korrekte' Lösung mit hohen Wiederholungszahlen eingeschliffen werden" (Schöllhorn, 2005, S. 127).

Dass Individualität und Variation im Techniktraining von besonderer Bedeutung sind, scheint für erfahrene Trainer und Trainerinnen jedoch keineswegs so neu zu sein, wie es von den Vertreter/innen des DL dargestellt wird. So verweist Killing (2009, S. 15) für das Leichtathletiktraining darauf, dass ,traditionelles Techniktraining“ eben „nicht auf Wiederholung [zielt], sondern auf Veränderung und Optimierung" und dass dabei bewusst Veränderungen genutzt werden, „im Hochsprung beispielsweise durch das Ändern der Anlaufwinkel, die Variation der Eingangsgeschwindigkeit, das bewusste Führen der Schwungelemente, einen greifend-schlagenden Fußaufsatz [oder] die Gewichtsentlastung."

Dass es sich hierbei nicht um eine bloße Einzelmeinung der Sportpraxis handelt, zeigt das von der Gruppe um Klaus Roth 1996 durchgeführte Interviewprojekt zum Techniktraining im Spitzensport. Die interviewten Trainerinnen und
Trainer maßen der Individualisierung des Trainings eine ganz hervorragende Bedeutung bei, sie konnte daher als eins von zehn Trainingsprinzipien herausgearbeitet werden. Als Beispiel sei hier die Auffassung von Wolfgang Maier, dem damaligen Damen-Bundestrainers im Riesenslalom, wörtlich wiedergegeben:

Für das Techniktraining geben Eckpfeiler (...) einen ,Würfel' vor, der von der Sportlerin nicht verlassen werden darf und der mit zunehmendem Niveau an Kantenlänge einbüßst. Innerhalb des Würfels werden individuelle (...) Lösungen als persönlicher Stil der Athletin zugelassen und diese Merkmale - anstelle einer Korrektur - gar gefördert. (Roth, 1996, S. 56)

Und in einer schriftlichen Befragung, die auf die qualitative Interviewphase des Projekts aufgesetzt wurde, stimmten immerhin $41 \%$ der befragten 152 deutschen Spitzentrainerinnen und -trainer folgender Aussage zu:

Ein Squashtrainer (...) ist nicht an einer korrekten Ausführung interessiert, sondern provoziert ungewöhnliche und auch ,falsche' Technikvarianten. Er definiert: Techniktraining ist für mich, daß die Spieler genau lernen, welche Bewegung unter welchen Bedingungen zu welchem Effekt führt. Können Sie dem - übertragen auf Ihre Sportart - zustimmen? (Roth 1996, S. 175)

Zumindest im Spitzenbereich ist es also keineswegs so, wie die Vertreter/-innen des DL nahe legen, dass ein ideales Technikleitbild unterschiedslos auf alle Athletinnen und Athleten angewandt und dann durch massive Wiederholung „eingeschliffen“ würde. Individualisierung und Variation spielen in der "traditionellen“ Sportpraxis vielmehr eine sehr große Rolle.

$\mathrm{Zu} \mathrm{c)} \mathrm{Wie} \mathrm{von} \mathrm{Killing} \mathrm{(2009)} \mathrm{anschau-}$ lich dargelegt, erscheint das Anpreisen des DL als neue, alleingültige Methode vor allem bei unerfahrenen Trainerinnen und Trainern problematisch, die ihr Wissen auf Fortbildungen aktualisieren. Als Ursache für den Erfolg des DL bei Trainerfortbildungen vermutet er das motivierende „Sonntagstraining“ sowie einen „verdeckten Methodenmix“. Wie Killing (2009) selbst beobachten konnte, wur- 
den in einer von Wolfgang Schöllhorn geleiteten Fortbildung zum DL die „zuvor kritisierten Lernmethoden (zielführende Übungsauswahl, -demonstration, -wiederholung, vereinzelte Korrekturen) eingesetzt“ (S. 13). All dies führe zwar zu einer begeisterten Zustimmung auf den Fortbildungen; Killing befürchtet aber, dass zunächst begeisterte Trainer/-innen auf längere Sicht enttäuscht werden, dies nicht zuletzt deshalb, weil „vielen Teilnehmern - im Unterschied zu den erfahrenen Referenten - die klaren Bewegungsvorstellungen und -räume [fehlten], um die herum bzw. in denen sie die einzelnen Trainingsaufgaben sinnvoll variieren bzw. differieren können“" (S. 12-13).

Dieser Befürchtung wollen wir uns anschließen, sie aber insofern ausweiten, als dass es dem DL ganz grundsätzlich an der exakten Definition der durchzuführenden Bewegungsvariationen mangelt und dieser Mangel dann notwendigerweise durch - mehr oder weniger - profundes Praxiswissen aufgefüllt werden muss. Insbesondere wollen wir diese Kritik bezogen wissen auf die im DL propagierte „Abtastung“ des „Lösungsraums“ vor allem „an den Rändern“ (z. B. Schöllhorn, 2005, S. 133), denn diese Forderung verlangt nach einer klaren Vorstellung davon, wo denn der Lösungsraum „aufhört" - und die hierzu notwendige Übersetzung der theoretischen Begrifflichkeiten in praktische Übungsformen ist alles andere als trivial. Ist es also beispielsweise tatsächlich so, dass das Spielen des Balles mit dem angewinkelten Oberarm als „chicken wing“, wie von Römer, Schöllhorn, Jaitner und Preiss (2009) als differenzielle Übungsvariation des VolleyballZuspielbaggers vorgeschlagen, tatsächlich noch zum Lösungsraum der Aufschlagannahme gehört? Und was ist auf der anderen Seite das Kriterium dafür, dass, wie von Schöllhorn, Beckmann, Janssen und Michelbrink (2009, S. 39) getan, „bewegungsferne Bewegungen wie Schwimmen, Radfahren etc. zum Erlernen des Kugelstoßens" ausgeschlossen werden, da bei Berücksichtigung solcher Variationen „die Lernfortschritte pro Zeit (...) wieder verringert [werden] “? Konkret gefragt: Ist z. B. der Tennis-Vorhand-Schlag zum Hockey-Vorhand-Schlag "bewegungsfern“ oder gehört er zu ein und demselben Lö- sungsraum, der sich dann wiederum auf welche Technik bezöge? Bevor Fragen dieser Art nicht klar und eindeutig von den Vertreter/-innen des DL beantwortet worden sind, handelt es sich beim DL um einen Praxisvorschlag mit sehr eingeschränkter Praktikabilität.

Man mag an dieser Stelle entgegnen, dass das Problem der exakten Definition von Verhaltens-, Handlungs- oder Motorikprogrammklassen eines ist, das nicht nur das DL betrifft. Und man mag in der Folge trefflich darüber streiten, ob beispielsweise die Abgrenzung der generalisierten motorischen Programme über die von Schmidt (1975) formulierten Invarianten nun sinnvoll ist oder nicht - auch wir haben an dieser Stelle unsere Zweifel. Aber immerhin: Man kann darüber streiten, weil die Invarianten in der Schematheorie wohl definiert sind, während im Konzept des DL genau diese Definition fehlt. Auf theoretischer Ebene macht dieses Fehlen den DL-Ansatz immun gegen Falsifizierungen, auf praktischer Ebene macht es den Ansatz beliebig.

\section{6. „Neue Kleider" mit fatalen Konsequenzen}

Fassen wir die bisher geübte Kritik zusammen, so erweist sich der Ansatz des DL als untauglich im Hinblick auf die Ableitung aus dem systemdynamischen Ansatz, auf die Abgrenzung gegenüber dem Informationsverarbeitungsansatz, auf den empirischen Wirksamkeitsnachweis sowie auf den Neuigkeitswert für die Sportpraxis. Eine Übersicht über die im Detail gesetzten Kritikpunkte zeigt $\bullet$ Tab. 1.

Zusammenfassend ergibt sich die folgende Beurteilung des hier kritisierten Ansatzes: Akzentuiert man die Forderungen des DL, sportliche Techniken unter randomisierten Bedingungen üben zu lassen, so entspricht dies voll und ganz sowohl der bestehenden Praxismeinung als auch den Praxisempfehlungen, die man in bewegungswissenschaftlichen Lehrbüchern unter Verweis auf das Kontextinterferenzparadigma findet. Akzentuiert man hingegen andere Aspekte des DL, so sind diese entweder objektiv falsch (z. B. die Forderung nach völligem Verzicht auf Rückmeldungen) oder weder theoretisch noch empirisch begründet (z. B. die For- derung der Maximierung von Differenzen zwischen unmittelbar aufeinander folgenden Übungen). Wie aufgezeigt wurde, reduziert sich damit der von den Vertreter/innen des DL geleistete Beitrag zur Sportwissenschaft darauf, für ein altbekanntes Phänomen ein neues Etikett gefunden und dieses extensiv in der Welt der Sportpraxis verbreitet $\mathrm{zu}$ haben.

Dies ist in zweierlei Hinsicht problematisch. So ist es zum ersten zu bedauern, wenn durch die blinde Übernahme des DL-Konzepts Lernende in ihrem Lernfortschritt behindert werden, etwa dadurch, dass ihnen hilfreiche Rückmeldungen vorenthalten werden und dass man sie beispielsweise in den evolutionär uralten Attraktor der geschlossenen Augen beim Blocken im Volleyball hineinfallen lässt, so dass sie dann in späteren Jahren erheblichen Aufwand betreiben müssen, sich dieses leistungshinderliche Ausführungsmerkmal wieder abzugewöhnen. Zum zweiten mögen - wie ausführlich von Killing (2009) dargestellt - Enttäuschungen auf Seiten der Lehrenden im Hinblick auf die versprochene Wirksamkeit des DL dazu führen, dass in Übergeneralisierung der negativen Erfahrungen die „Leistungen [der Forscher] zunehmend skeptisch, wenn nicht sogar ablehnend aufgenommen werden" (S. 15). Wir teilen diese Befürchtungen, unter anderem sind sie die Motivation für diesen Beitrag.

\section{Verantwortung und Hoffnungen für die Zukunft}

Natürlich ist uns bewusst, dass die hier geübte Kritik harsch ausfällt, und ebenso, dass Ideen immer auch mit Personen verbunden sind, hier insbesondere: mit Wolfgang Schöllhorn als Urheber des DL. Unsere an der Sache orientierte Kritik des differenziellen Lehrens und Lernens ist somit auch unausweichlich eine Kritik an der wissenschaftlichen Arbeit von Wolfgang Schöllhorn und seinen Mitarbeiter/ -innen und könnte so als persönliche Kritik missverstanden werden. Unser hiermit verbundenes Unbehagen wird erstens dadurch gemildert, dass wir mit dieser Kritik einer Verantwortung gegenüber der Sportpraxis und der eigenen Wissenschaftsdisziplin, denen wir uns verpflichtet fühlen, nachkommen. Zweitens wird 
Tab. 1 Kritikpunkte am differenziellen Lernen (DL) im Überblick

(1) DL lässt sich nicht aus dem systemdynamischen Ansatz ableiten. Kognitivismus ist lücken- und fehlerhaft.

(3) Der Nachweis der Wirksamkeit des DL wird empirisch nicht erbracht.

(4) Der Sportpraxis liefert das DL nichts Neues. Idealbewegungen. „eingeschliffen“ werden sollten. völlig unberücksichtigt. reichend referiert. formen Regeln interpretiert. wissenschaftlichen Standard. Widersprüche auf. nicht einer Leitbildorientierung.
(2) Die Abgrenzung des DL zum

(a) Bewegungslernen entspricht nicht dem Auffinden eines globalen Minimums.

(b) Der systemdynamische Ansatz ist neutral gegenüber Empfehlungen zur Rückmeldungsgabe.

(c) Verstärkte Streuungen führen nicht zu Veränderungen der systemischen Potenziallandschaft.

(d) Ein Einkreisen der individuellen Optimallösung ist in hochdimensionalen Räumen unmöglich.

(a) Motorikprogramme beziehen sich nicht auf definierte

(b) Aus Programmtheorien folgt nicht, dass Bewegungen

(c) Der themenrelevante Kontextinterferenzansatz wird zuerst ignoriert und dann fehlinterpretiert.

(d) Themenrelevante Arbeiten zur Rückmeldungsgabe bleiben

(e) Themenrelevante Arbeiten zur Variabilität werden unzu-

(f) Jüngere Entwicklungen nach Ende der Motor-action-Kontroverse wurden vollständig verpasst.

(a) Das gewählte experimentelle Design erlaubt nicht den Ausschluss alternativer Erklärungen.

(b) Empirische Befunde werden nicht nach wissenschaftskon-

(c) Die statistische Auswertung der Daten entspricht nicht dem

(d) Berichtete und tatsächliche empirische Befundlage weisen

(a) Die Nichtwiederholbarkeit von Bewegungen widerspricht

(b) Individualität und Variation finden in der Sportpraxis bereits umfassende Berücksichtigung.

(c) Unzureichende Definitionen der Bewegungsvariationen führen zu mangelnder Praktikabilität.

das Gewissen auch durch die Erwartung etwas beruhigt, dass Kollegen, die Kritik eines ausgewiesenen, allerdings "nur“ promovierten Sportpraktikers als „AnmaBung" eines ,wissenschaftlich Qualifizierten“ gegenüber ,wissenschaftlich Hochqualifizierten" abtun (Schöllhorn, Beckmann \& Janssen, 2009), sicherlich selbst eine harsche Entgegnung vertragen können. Und drittens wollen wir - in Erinnerung an das Kopfzitat zu diesem Beitrag - die Hoffnung nicht begraben, dass das wirkliche Leben mitunter märchenhaft ausfällt. Wie uns Andersen zeigt, mag Einsicht auch von vorübergehend Verblendeten letztlich als Gewinn erlebt werden; allein scheint es des desillusionierenden Anstoßes zu bedürfen. Als genau
Open Access. This article is distributed under the terms of the Creative Commons Attribution License which permits any use, distribution, and reproduction in any medium, provided the original author(s) and the source are credited.
Interessenskonflikt. Der korrespondierende Autor gibt für sich und seinen Koautor an, dass kein Interessenskonflikt besteht.

\section{Literatur}

1. Adams, J.A. (1971). A closed-loop theory of motor learning. Journal of Motor Behavior, 3, 111-150.

2. Beckmann, H. \& Schöllhorn, W. I. (2006). Differenzielles Lernen im Kugelstoßen. Leistungssport, 36 44-50.

3. Davids, K., Button, C. \& Bennett, S. (2008). Dynamics of skill acquisition: $A$ constraints-led approach. Champaign/IL: Human Kinetics.

4. Haken, H., Kelso, J. A. S. \& Bunz, H. (1985). A theoretical model of phase transitions in human hand movements. Biological Cybernetics, 51, 347-356.

5. Hoffmann, J. (1993). Vorhersage und Erkenntnis. Göttingen: Hogrefe.

6. Hohmann, A., Lames, M. \& Letzelter, M. (2007). Einführung in die Trainingswissenschaft (4. Aufl.). Wiebelsheim: Limpert.

7. Hossner, E.-J. \& Künzell, S. (2003). Motorisches Lernen. In H. Mechling \& J. Munzert (Hrsg.), Handbuch Bewegungswissenschaft - Bewegungslehre (S. 131-153). Schorndorf: Hofmann.

8. Kelso, J.A.S. (1995). Dynamic patterns. Self-organization of brain and behavior. London: MITPress.

9. Killing, W. (2009). Kritik der "Praxis" an der Sportwissenschaft. leichtathletiktraining, o.J. (1), 12-15.

10. Kostrubiec, V., Tallet, J. \& Zanone, P. G. (2006). How a new behavioral pattern is stabilized with learning determines its persistence and flexibility in memory. Experimental Brain Research, 170, 238244.

11. Lashley, K. S. (1917). The accuracy of movement in the absence of excitation from the moving organ. American Journal of Physiology, 43, 169-194.

12. Lee, T. D. \& Magill, R. A. (1983). The locus of contextual interference in motor-skill acquisition. Journal of Experimental Psychology: Learning, Memory and Cognition, 9, 730-746.

13. Magill, R. A. (1998). Motor learning. Concepts and applications (5. Aufl.). Boston/MA: McGraw Hill.

14. Magill, R. A. \& Hall, K. G. (1990). A review of the contextual interference effect in motor skill acquisition. Human Movement Science, 9, 241-289.

15. Marschall, F. \& Daugs, R. (2003). Feedback. In H. Mechling \& J. Munzert (Hrsg.), Handbuch Bewegungswissenschaft - Bewegungslehre (S. 281294). Schorndorf: Hofmann.

16. Meijer, O. G. \& Roth, K. (Hrsg.) (1988). Complex movement behaviour: "The" motor action controversy. Amsterdam: North-Holland/Elsevier.

17. Müller, H. (2001). Ausführungsvariabilität und Ergebniskonstanz. Lengerich: Pabst.

18. Pieck, J. P. (Hrsg.) (1998). Motor behavior and human skill. A multidisciplinary approach. Champaign/IL: Human Kinetics.

19. Rehers, D., Beckmann, H. \& Schöllhorn, W. (2006). Differenzielles Lernen im Fußball am Beispiel des Dribbelns. In B. Halberschmidt \& B. Strauß (Hrsg.), Elf Freunde sollt ihr sein!? (S. 114). Hamburg: Czwalina.

20. Römer, J., Schöllhorn, W. I., Jaitner, T. \& Preiss, R. (2003). Differenzielles Lernen bei der Aufschlagannahme im Volleyball. In J. Krug \& T. Müller (Hrsg.), Messplätze, Messplatztraining, Motorisches Lernen (S. 129-133). Sankt Augustin: Academia. 
21. Römer, J., Schöllhorn, W. I., Jaintner, T. \& Preiss, R. (2009). Differenzielles Lernen im Volleyball. Ein Unterrichtsvorhaben zur Verbesserung der Annahme. sportunterricht, 58 (2), 41-45.

22. Rosenbaum, D. A., Kenny, S. \& Derr, M. A. (1983). Hierarchical control of rapid movement sequences. Journal of Experimental Psychology: Human Perception and Performance, 16, 439-444.

23. Roth, K. (Hrsg.) (1996). Techniktraining im Spitzensport. Köln: Sport und Buch Strauß.

24. Roth, K. \& Willimczik, K. (Hrsg.) (1999). Bewegungswissenschaft. Reinbek: Rowohlt.

25. Savelsbergh, G. J. P., Kamper, W., Rabius, J., De Koning, J. \& Schöllhorn, W. (2010). A new method to learn to start in speed skating: A differencial learning approach. International Journal of Sport Psychology, 41, 415-427.

26. Schmidt, R. A. (1975). A schema theory of discrete motor skill learning. Psychological Review, 82, 229-261.

27. Schmidt, R. A. (1991). Frequent augmented feedback can degrade learning: Evidence and interpretations. In G. E. Stelmach \& J. Requin (Hrsg.), Tutorials in Motor Neuroscience (S. 59-75). Dordrecht: Kluwer.

28. Schöllhorn, W. I. (1999). Individualität - ein vernachlässigter Parameter? Leistungssport, 29 (2), 512.

29. Schöllhorn, W. I. (2000). Applications of system dynamic principles to technique and strength training. Acta Academiae Qlympiquae Estoniae, 8, 67-85.

30. Schöllhorn, W. I. (2005). Differenzielles Lehren und Lernen von Bewegungen. Durch veränderte Annahmen zu neuen Konsequenzen. In H. Gabler, $\mathrm{U}$. Göhner \& J. Schiebl (Hrsg.), Zur Vernetzung von Forschung und Lehre in Biomechanik, Sportmotorik und Trainingswissenschaft (S. 125-135). Hamburg: Czwalina.

31. Schöllhorn, W. I. (2010), Differenzielles Lernen im Schwimmen - eine Alternative? In Deutsche Schwimmtrainer-Vereinigung (Hrsg.), Schwimmen - Lernen und Optimieren, Bd. 31 (S. 7-22). Wuppertal: Eigenverlag.

32. Schöllhorn, W. I., Beckmann, H. \& Janssen, D. (2009). Unsere Trainer sind sehr wohl mündig. Gegendarstellung zu einem Beitrag von W. Killing in Leichtathletiktraining $1 / 09$. Zugriff am 22. November 2011 unter http://www.sport.uni-mainz. de/674.php

33. Schöllhorn, W. I., Beckmann, H., Janssen, D. \& Drepper, J. (2010). Stochastic perturbations in athletics field events enhance skill acquisition. In I. Renshaw, K. Davids \& G. J. P. Savelsbergh (Hrsg.), Motor learning in practice. A contraints-led approach (S. 69-82). London: Routledge.

34. Schöllhorn, W. I., Beckmann, H., Janssen, D. \& Michelbrink, M. (2009). Differenzielles Lehren und Lernen im Sport. Ein alternativer Ansatz für einen effektiven Schulsportunterricht. sportunterricht, $58(2), 36-40$.

35. Schöllhorn, W. I. \& Köpsel, M. (2005). Differenzielles Lernen im Tischtennis. Teil 1: Kick it like Waldner. Verband Deutscher Tischtennistrainer, Trainerbrief, o.J. (4), 4-11.

36. Schöllhorn, W. I. \& Paschke, M. (2007). Differenzielles Training. Volleyballmagazin, 12, 28-36.

37. Schöllhorn, W. I., Paschke, M. \& Beckmann, H. (2006). Differenzielles Training im Volleyball beim Erlernen von zwei Techniken. In K. Langolf \& R. Roth (Hrsg.), Volleyball 2005 - Beach-WM (S. 97105). Hamburg: Czwalina.
38. Schöllhorn, W. I., Sechelmann, M., Trockel, M. \& Westers, R. (2004). Nie das Richtige trainieren, um richtig zu spielen. Leistungssport, 34 (5), 13-17.

39. Schöner, G. \& Kelso, J. A. S. (1988). A dynamic pattern theory of behavioral change. Journal of Theoretical Biology, 135, 501-524.

40. Shea, J. B. \& Morgan, R. L. (1979). Contextual interference effects on the acquisition, retention, and transfer of a motor skill. Journal of Experimental Psychology: Human Learning and Memory, 5 , 179-187.

41. Shea, C. H. \& Wulf, G. (2005). Schema theory: A critical appraisal and reevaluation. Journal of Motor Behavior, 37, 85-101.

42. Tallet, J., Kostrubiec, V. \& Zanone, P. G. (2008). The role of stability in the dynamics of learning, memorizing, and forgetting new coordination patterns. Journal of Motor Behavior, 40, 103-116.

43. Tallet, J., Kostrubiec, V. \& Zanone, P. G. (2010). Proactive transfer of learning depends on the evolution of prior learned task in memory. Human Movement Science, 29, 349-368.

44. Trockel, M. (2002). Differenzielles Torschusstraining im Fußball. Unveröffentlichte Staatsexamensarbeit, Universität Münster.

45. Trockel, M. \& Schöllhorn, W. I. (2003). Differenzielles Torschusstraining im Fußball. In J. Krug \& T. Müller (Hrsg.), Messplätze, Messplatztraining, Motorisches Lernen (S. 102-107). Sankt Augustin: Academia.

46. Van Rossum, J. (1990). Schmidt's schema theory. The empirical base of the variability of practice hypothesis: A critical analysis. Human Movement Science, 9, 387-435.

47. Winstein, C. J. \& Schmidt, R. A. (1990). Reduced frequency of knowledge of results enhances motor skill learning. Journal of Experimental Psychology: Learning, Memory and Cognition, 16, 677691.

48. Wulf, G. (1994). Zur Optimierung motorischer Lernprozesse. Untersuchungen zur Funktion von Kontext-Interferenz und Rückmeldungen beim Erwerb generalisierter motorischer Programme und motorischer Schemata. Schorndorf: Hofmann.

49. Zanone, P. G. \& Kelso, J. A. S. (1992). Evolution of behavioral attractors with learning: Nonequilibrium phase transitions. Journal of Experimental Psychology: Human Perception and Performance, 18, 403-421.

50. Zanone, P. G., Kostrubiec, V., Albaret, J. M. \& Temprado, J. J. (2010). Covariation of attentional cost and stability provides further evidence for two routes to learning new coordination patterns. Acta Psychologica, 133, 107-118. 